\title{
Performance Evaluation of Naval Minesweeping and Estimation of the Number of Remaining Mines based on Bayesian Estimation Theory
}

\author{
Xinqin Chen ${ }^{1 a^{*}}$, Huidong $\mathrm{Lu}^{1}$, Longteng Li, Chang Shu${ }^{1}$, Xuanmin $\mathrm{Li}^{2}$, \\ Chengwen Zhu ${ }^{1}$,XianYu Meng ${ }^{1}$,Zhang Tian ${ }^{1}$ \\ ${ }^{I}$ Dept. of Command and control, Wuhan Ordnance Officer School, Wuhan, Hubei, 430075, China \\ ${ }^{2}$ Dept. of Computer, Wuhan Bridge College, Wuhan, Hubei, 430052, China \\ acxqing100@sohu.coml
}

Keywords: Bayesian estimation mine sweeping probability remaining mine.

Abstract: It is very difficult to get exact accurate estimated value When the Markov process theory is used to calculate the number of remaining mines, because the prior knowledge has not been fully utilized. For solving the above problems, this paper establishes posterior distribution model of Estimation of the number of remaining mines, based on Bayesian estimation theory. Then several typical distributions are discussed, such as the binomial distribution, Poisson distribution, negative binomial distribution and uniform distribution, and the mean and variance of the number of remaining mines are given. Finally the simulation calculation is carried out for the given minefield prior data, the results show that the model accuracy is related to mine number, mine sweeping probability and other factors.

\section{Introduction}

Using Markov process to estimate the remaining number of mine is a common method [1-5], but it is difficult to get accurate result. Bayesian estimation method [6-8], which can make full use of prior knowledge and obtain high estimation precision, has been one of the hot research topic in recent years [8-10]. Minesweeping effect evaluation method based on Bayesian estimation theory is a mathematical statistical inference on the minefield state. It involves overall information of mine, sampling information and prior information, etc. In this paper, the minesweeping effect on the number of remaining mines is studied by using the theory of Bayesian estimation, especially for the different DC[1] and mixed layout minefield. Firstly, we make comprehensive analysis on the prior information of minefield mine number and quantify the formation to build the prior distribution model, then combine the constrained mine number with the sampling information of mine, finally take the posteriori estimation on remaining mines.

\section{Mathematical Model based on Bayesian estimation theory}

If the total number of mine is $N$, the prior distribution of mine is $\pi(m)$, the minefield is divided into $\mathrm{n}$ regions, the initial allocation proportion of the mine number is $\mathbf{m}_{0}=\left[m_{10}, m_{20}, \mathrm{~L}, m_{n 0}\right]$. When the $j$ th estimation is done, the mine sweeping probability of the single mine in the sub area is $\mathbf{p}_{j}=\left[p_{1 j}, p_{2 j}, \mathrm{~L} p_{n j}\right]^{T}$, the number of mines in each sub region between $\mathrm{j}$ and $\mathrm{j}-1$ is $\mathbf{x}_{j}=\left[x_{1 j}, x_{2 j}, \mathrm{~L} x_{n j}\right]$.

Suppose $\mathbf{X}_{j}=\left[\mathbf{x}_{1}, \mathbf{x}_{2}, \mathrm{~L} \mathbf{x}_{j}\right]^{T}, N_{j}=\sum_{k=1}^{j} \sum_{i=1}^{n} x_{i j}, \Delta N_{j}=N_{j}-N_{j-1}, P_{j}=\mathbf{m}_{0} \times \mathbf{p}_{j}$

According to Bayesian statistical theory, when the remaining number of mine is estimated for the first time, the joint distribution of the sample and the number of remaining mines of the whole area is:

$$
h\left(R_{1}, \mathbf{X}_{1}\right)=\pi\left(R_{1}+N_{1}\right)\left(\begin{array}{c}
R_{1}+N_{1} \\
N_{1}
\end{array}\right) P_{1}^{N_{1}}\left[1-P_{1}\right]^{R_{1}}
$$


Under the condition of the $\mathbf{X}_{1}$, the conditional distribution of $R_{1}$ is:

$$
\pi\left(R_{1} \mid \mathbf{X}_{1}\right)=\pi\left(R_{1}+N_{1}\right) \frac{\left(R_{1}+N_{1}\right) !}{R_{1} !}\left[1-P_{1}\right]^{R_{1}} /\left[\sum_{R_{1}=0}^{N-N_{1}} \pi\left(R_{1}+N_{1}\right) \frac{\left(R_{1}+N_{1}\right) !}{R_{1} !}\left[1-P_{1}\right]^{R_{1}}\right]
$$

For each sub area, when the number of remaining mine is estimated for the first time, the distribution proportion of the number of remaining mines in sub area $i$ will become:

$$
m_{i 1}=m_{i 0} \frac{1-p_{i 1}}{1-P_{1}}
$$

The posterior distribution of the number of remaining mines can be obtained as follows:

$$
\pi\left(r_{i 1} \mid \mathbf{X}_{1}\right)=\sum_{R_{1}=r_{i 1}}^{N-N_{1}} \pi\left(R_{1} \mid \mathbf{X}_{1}\right)\left(\begin{array}{l}
R_{1} \\
r_{i 1}
\end{array}\right)\left(m_{i 1}\right)^{r_{i 1}}\left[1-m_{i 1}\right]^{R_{1}-r_{i 1}}
$$

If $G_{1}=\sum_{R_{1}=0}^{N-N_{1}} \pi\left(R_{1}+N_{1}\right) \frac{\left(R_{1}+N_{1}\right) !}{N_{1} !}\left[1-P_{1}\right]^{R_{1}}$, the above expression can be rewritten as follows:

$$
\begin{aligned}
\pi\left(r_{i 1} \mid \mathbf{X}_{1}\right) & =\frac{1}{G_{1}} \sum_{R_{1}=r_{11}}^{N-N_{1}}\left\{\pi\left(R_{1}+N_{1}\right) \frac{\left(R_{1}+N_{1}\right) !}{R_{1} !} \frac{R_{1} !}{\left(R_{1}-r_{i 1}\right) ! r_{i 1} !}\left[1-P_{1}\right]^{R_{1}}\left(m_{i 0} \frac{1-p_{i 1}}{1-P_{1}}\right)^{r_{i 1}}\left[1-m_{i 0} \frac{1-p_{i 1}}{1-P_{1}}\right]^{R_{1}-r_{i 1}}\right\} \\
= & \frac{1}{G_{1}} \sum_{R_{1}=r_{i 1}}^{N-N_{1}} \pi\left(R_{1}+N_{1}\right) \frac{\left(R_{1}+N_{1}\right) !}{\left(R_{1}-r_{i 1}\right) ! r_{i 1} !} \cdot\left[m_{i 0}\left(1-p_{i 1}\right)\right]^{r_{i 1}}\left[1-P_{1}-m_{i 0}\left(1-p_{i 1}\right)\right]^{R_{1}-r_{i 1}}
\end{aligned}
$$

The $\pi\left(R_{1} \mid \mathbf{X}_{1}\right)$ can be seen as the prior distribution of the whole area in the second estimation. The mine detection probability between the 1 st estimation and the 2 nd estimation can be stated as follows:

$$
p_{i 1}=\frac{p_{i 2}-p_{i 1}}{1-p_{i 1}}(6)
$$

\section{Suppose}

$$
P_{1}=\left[m_{11}, m_{21}, \mathrm{~L}, m_{n 1}\right]\left[p_{1 j}, p_{2 j}, \mathrm{~L} p_{n j}\right]^{T}=\left(P_{2}-P_{1}\right) /\left(1-P_{1}\right)
$$

Then the joint distribution of remaining mine number $R_{2}$ and sample $\mathbf{X}_{2}$ can be obtained:

$$
h\left(R_{2}, \mathbf{X}_{2}\right)=\pi\left(R_{2}+\Delta N_{2} \mid \mathbf{X}_{1}\right)\left(\begin{array}{c}
R_{2}+\Delta N_{2} \\
\Delta N_{21}
\end{array}\right) P_{1}^{\Delta N_{2}}\left[1-P_{1}\right]^{R_{2}} \pi\left(R_{2}+N_{2}\right) \frac{\left(R_{2}+\Delta N_{2}\right)}{R_{2} ! \Delta N_{2} !}\left(P_{2}-P_{1}\right)^{\Delta N_{2}}\left[1-P_{2}\right]^{R_{2}}
$$

So the posterior distribution of $R_{2}$ is:

$$
\begin{aligned}
\pi\left(R_{2} \mid \mathbf{X}_{2}\right) & =\frac{1}{G_{2}} \pi\left(R_{2}+N_{2}\right) \frac{\left(R_{2}+N_{2}\right) !}{R_{2} !}\left[1-P_{2}\right]^{R 2} \\
\text { Where } G_{2} & =\sum_{R_{2}=0}^{N-N_{2}} \pi\left(R_{2}+N_{2}\right) \frac{\left(R_{2}+N_{2}\right) !}{N_{2} !}\left[1-P_{2}\right]^{R_{2}}
\end{aligned}
$$

Similarly, the posterior distribution of remaining mines in sub region $i$ can be obtained as follows:

$$
\pi\left(r_{i 2} \mid \mathbf{X}_{2}\right)=\frac{1}{G_{1}} \sum_{R_{2}=r_{i 2}}^{N-N_{2}} \pi\left(R_{2}+N_{2}\right) \frac{\left(R_{2}+N_{2}\right) !}{\left(R_{2}-r_{i 2}\right) ! r_{i 2} !}\left[m_{i 0}\left(1-p_{i 2}\right)\right]^{r_{i 2}}\left[1-P_{2}-m_{i 0}\left(1-p_{i 2}\right)\right]^{R_{2}-r_{i 2}}
$$

The distribution of remaining number of mines that belongs to the $j$ th estimation can be obtained: 


$$
\left\{\begin{array}{l}
\pi\left(R_{j} \mid \mathbf{X}_{j}\right)=\frac{1}{G_{j}} \pi\left(R_{j}+N_{j}\right) \frac{\left(R_{j}+N_{j}\right) !}{R_{j} !}\left[1-P_{j}\right]^{R_{j}} \\
\pi\left(r_{i j} \mid \mathbf{X}_{j}\right)=\frac{1}{G_{1}} \sum_{R_{j}=r_{i j}}^{N-N_{j}} \pi\left(R_{j}+N_{j}\right) \frac{\left(R_{j}+N_{j}\right) !}{\left(R_{j}-r_{i j}\right) ! r_{i j} !}\left[m_{i 0}\left(1-p_{i j}\right)\right]^{r_{i j}}\left[1-P_{j}-m_{i 0}\left(1-p_{i j}\right)\right]^{R_{j}-r_{i j}} \\
G_{j}=\sum_{R_{j}=0}^{N-N_{j}} \pi\left(R_{j}+N_{j}\right) \frac{\left(R_{j}+N_{j}\right) !}{N_{j} !}\left[1-P_{j}\right]^{R_{j}}
\end{array}\right.
$$

By analysis of the above expression, it can be found that the distribution of remaining number of mines is only related to the swept mines, mine sweeping probability and initial state of mines, which has little to do with previous estimation. So the above expression can be rewritten as follows:

$$
\left\{\begin{array}{l}
\pi(R \mid \mathbf{X})=\frac{1}{G} \pi(R+N) \frac{(R+N) !}{R !}[1-P]^{R} \\
\pi\left(r_{i} \mid \mathbf{X}\right)=\frac{1}{G} \sum_{R=r_{i}}^{N-N_{f}} \pi(R+N) \frac{(R+N) !}{\left(R-r_{i}\right) ! r_{i} !}\left[m_{i 0}\left(1-p_{i}\right)\right]^{r_{i}}\left[1-P-m_{i 0}\left(1-p_{i}\right)\right]^{R-r_{i}} \\
G=\sum_{R_{j}=0}^{N-N_{j}} \pi(R+N) \frac{(R+N) !}{N !}[1-P]^{R}
\end{array}\right.
$$

Where $R$ is the remaining number of mines of the current status, is the sample of swept mines in the current status, $N$ is the total number of mines, $N_{f}$ is the total number of swept mines.According to the above expression, the posterior distribution the number of remaining mines can be obtained, and then the mean and interval estimation of remaining mines can be figured out.

Assume that the largest number of mines which have been laid is $N$, the mines that have been found is $N_{f}$. According to the posterior distribution for remaining mines $\pi(R \mid \mathbf{X})$, the mean and variance of remaining mines can be obtained:

$$
\left\{\begin{array}{l}
E_{R}=\sum_{R=0}^{N-N_{f}} \pi\left(R \mid N_{f}\right) \cdot R \\
V_{R}=\sum_{R=0}^{N-N_{f}} \pi\left(R \mid N_{f}\right) \cdot\left(R-E_{R}\right)^{2}
\end{array}\right.
$$

\section{Emulation and results}

For comparing the estimated results with the true value of remaining mine number, as well as to extract swept mine samples from calculation model, this paper takes a simple simulation.

Generate a random number $p_{i}(0 \sim 1)$ for each mine, if $p_{i}$ is less than the threshold valve $P_{0}^{\prime}$, then the mine is seen as a swept mine. Simulate the mine sweeping process for all the mines, quantify the number of swept mines, and take it as the true value of the process.

Assume that there are 60 mines in one minefield and the maximum value of $D C$ is 4 . According to different, its distribution is $[25 ; 15 ; 15 ; 5]$. Substitute the minesweeping probability (0.6) into the foregoing model, then take the emulation 60 rounds ( $\mathrm{n}$ times per round).

The estimated values of the remaining mines in above mine field are plotted in Fig. 1, When $n=4$ : 

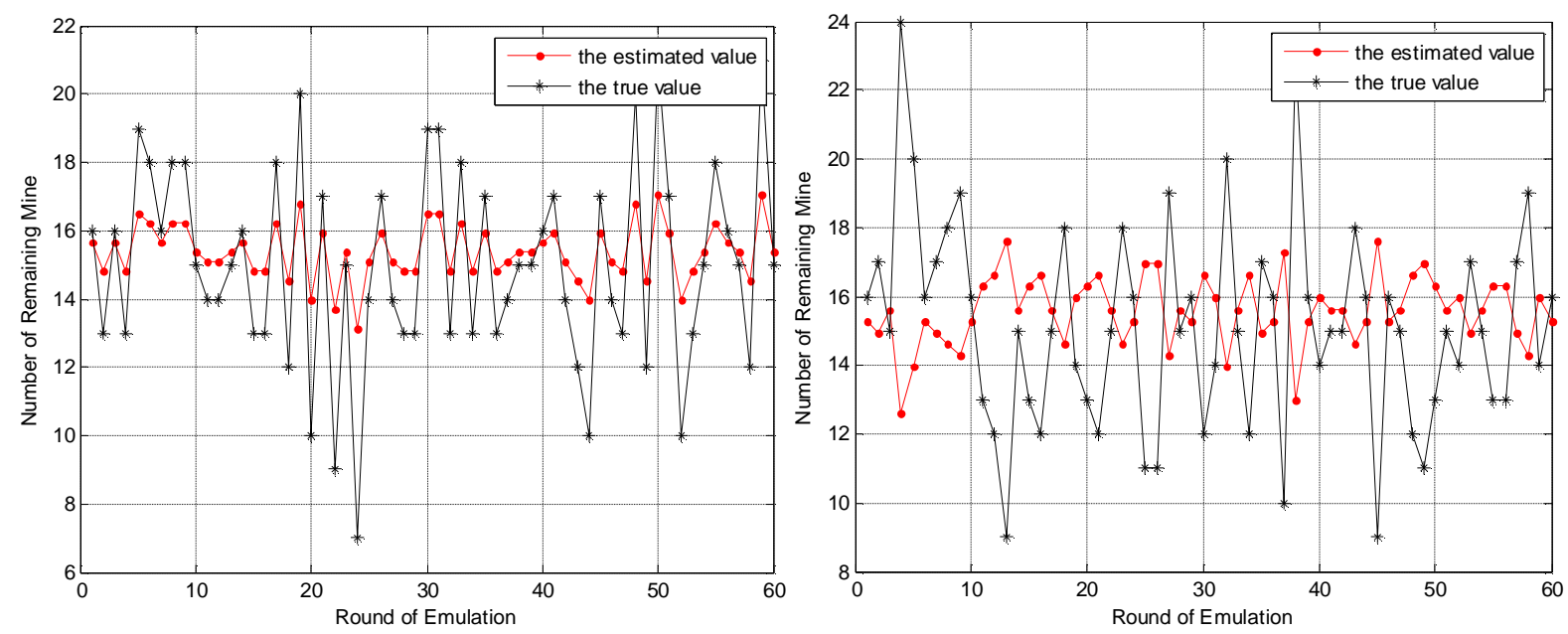

Fig. 1a The result of binomial distribution

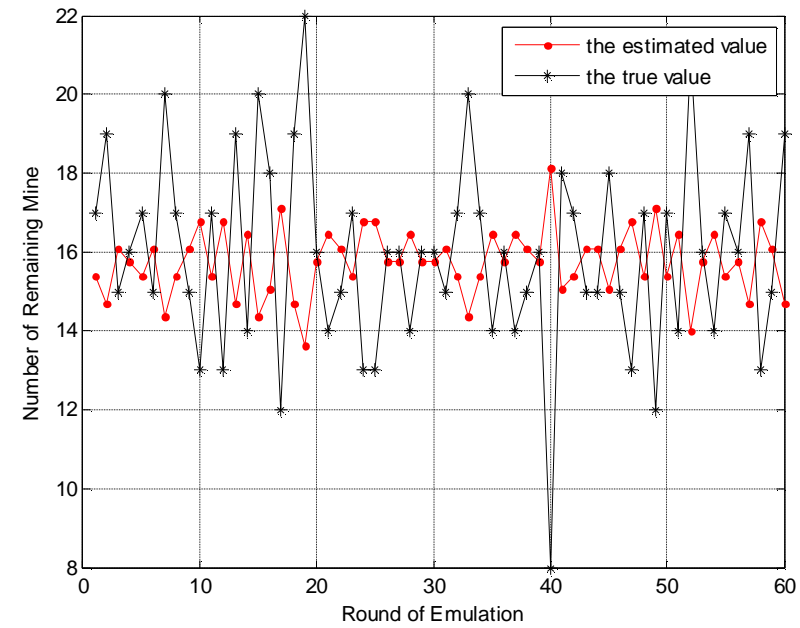

Fig. 1c The result of uniform distribution

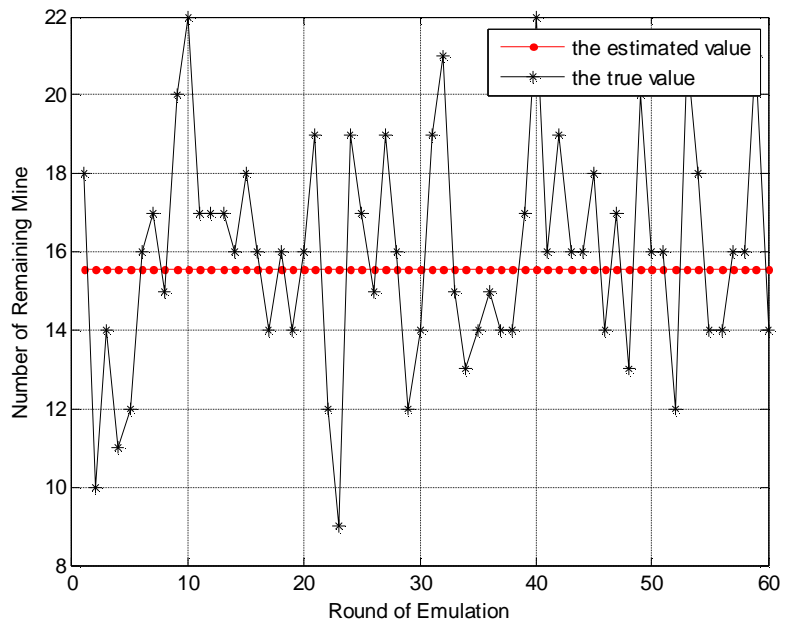

Fig. 1d The result of Poisson distribution

In Fig. 1, the binomial distribution is $B(100,0.60)$, the uniform distribution is $U(40,80)$. If we change the parameters to make a prior information closer to the truth, for example, take $B(80,0.75)$ and $U(50,70)$ as the prior distribution, then the estimation results of remaining mines can be obtained as Fig. 2:

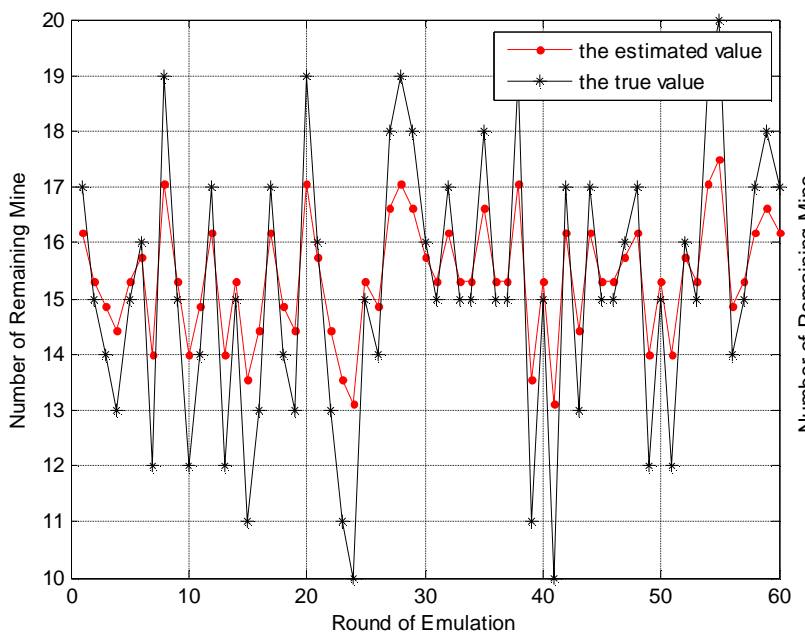

Fig. 2a The result of prior distribution with $B(80,0.75)$

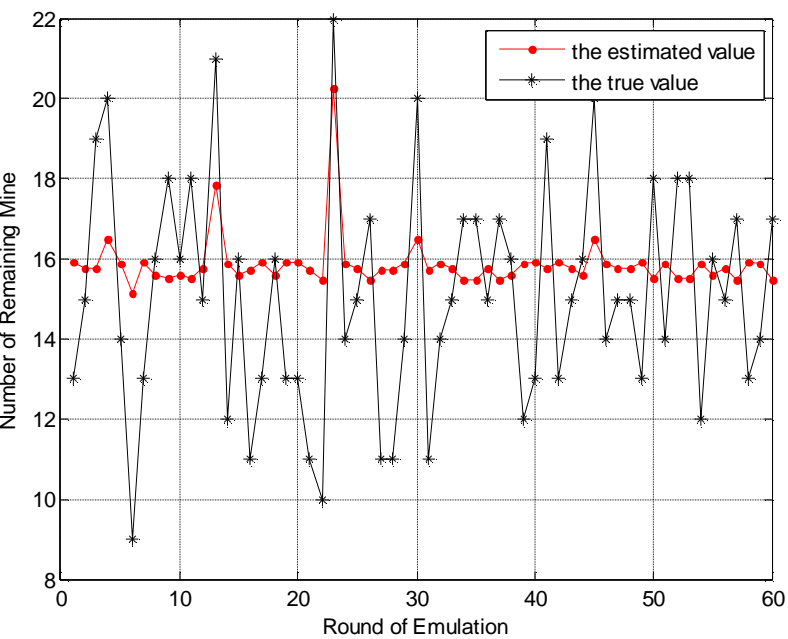

Fig. $2 b$ The result of prior distribution with $U(50,70)$ 


\section{Discussions and Conclusions}

We can find that the estimation results of remaining mines subject to prior distribution in change rules in fig. 1. If we take binomial distribution as the prior distribution, the change tendency of the posterior mean value and true value is consistent basically. For negative binomial distribution and uniform distribution, the change tendency of the posterior mean value and true value is opposite, but the extent of the reverse change is small due to the restraint of a prior information. As for Poisson distribution, it's posterior mean value hardly has any change at all.

By analysis of fig. 1, the binomial distribution, Poisson distribution and negative binomial distribution, correspond to the quality of different prior information of mines respectively: high, middle and low. For accurate priori information, use the binomial distribution, then choose Poisson distribution, once again choose negative binomial distribution. If having some knowledge of mine number range, choose uniform distribution.

In fig. 2a, we can find that the amplitude of the posterior estimate has been greatly improved for binomial distribution. Compared the situation shown in Fig. 1a, the amplitude difference is much smaller. For the amplitude of uniform distribution, its opposite trend started to change to the same trends. This shows that when we use the Bayesian method to estimate the number of remaining mines, a prior accuracy of the information will play a crucial role.

\section{References}

[1] Chen Xin-qin, Lu Hui-dong, Zhu Cheng-wen.Performance Evaluation of Naval Minesweeping and Estimation of the Number of Remaining Mines Based on Markov Process. $\operatorname{ETEEEM(2014):739-742~}$

[2] Chen Xin-qin, Li Longteng, Li Xuanmin.Research on the evaluation model of effect of naval minesweeping operation in complex conditions. ETEEEM (2014):743-747

[3] Chen Xin-qin. Study on Pivotal Techniques of Mine Sweeping Based on Magnetic Fuse Mine [D]. Wuhan: Naval University of Engineering. 2009.

[4] Chen Xin-qin, Lin Chun-sheng, Zhang yong-zhi.Studies of Carlo Simulations and Markov process to Estimating Effect of Naval Mine-sweeping [J]. Journal of WuhanUniversity of Technology (Transportation Science \& Engineering), March (3). 2010.

[5] Zhang xu. Study on the operation of anti mine warfare for mine-hunting ship [D]. Dalian : Dalian Naval Academy Press. 2005.

[6] Wu Xi-zhi.Bayesian statistics [M]. Beijing: China Statistics Press, 2000.

[7] Jia Nai-guang.Statistical Decision deals with Bayesian analysis. Beijing: China Statistics Press , 2001.

[8] Khalaf-Allah M , Kyamakya K , Mobile Location in GSM Networks using Database Correlation with Bayesian Estimation , Computers and Communications[C]. 11th IEEE Symposium on Computers and Communications , 2006:289-293.

[9] JilkovV P , Li X R. Online Bayesian estimation of transition probabilities for Markovian jump systems[C]. IEEE Transactions on Signal Processing , 2004:1620-1630.

[10] Bryan K , Carthel C. A Bayesian approach to predicting an unknown number of targets based on sensor performance [C]. 9th International Conference on Information Fusion, 2006:1-7. 